\title{
ARON GURWITSCH: \\ FENOMENOLOGÍA DE LA PERCEPCIÓN \\ Y ENCUENTRO CON KURT GOLDSTEIN
}

\author{
María da Luz Pintos-Peñaranda \\ Universidade de Santiago de Compostela'
}

Aron Gurwitsch, fenomenólogo de origen lituano, nacido en 1901, entra en Francia en abril de 1933 como refugiado judío procedente de Alemania, en donde había realizado sus estudios y en donde se había doctorado. En el momento de entrar en Francia tenía treinta y dos años. Cinco años atrás había presentado su Tesis Doctoral. A los pocos meses de llegar a París comienza a impartir sus cursos de introducción sistemática a la fenomenología en la Universidad de la Sorbona y, acerca de esto, es muy importante que conozcamos y reconozcamos - que es él el primero que hace en Francia, como fenomenólogo, una exposición profunda y sistemática de la fenomenología husserliana, ya que hasta ese momento tan sólo había habido unas conferencias (de Georges Gurvitch), tres libros sobre Husserl (uno de Jean Héring, ${ }^{2} 0$ otro de Emmanuel Lévinas ${ }^{3}$ y otro de Georges Gurvitch), ${ }^{4}$ y alguna que otra breve recensión

I asmaluri@usc.es

2 Phénoménologie et Philosophie religieuse, Paris, Faculté de Théologie Protestante de l'Université de Strasbourg, 1926.

3 El libro de Emmanuel Lévinas La théorie de l'intuition dans la phénoménologie de Husserl, Paris, Vrin, 1930.

Por tanto, con tan sólo estos tres textos, el de J. Héring, el de E. Lévinas y el de Georges Gurvitch, es evidente que hasta que Aron Gurwitsch da sus cursos de fenomenologia era muy escasa la información habida en Francia hasta ese momento sobre esta nueva filosofia.

4 En 1928, 1929 y 1930, Georges Gurvitch impartió unos cursos libres en la Universidad de la Sorbona. Un extracto de la parte dedicada a Husserl fue publicado por él en Revue de Métaphysique et de Morale y ya en el año 1930 publicó el conjunto de estos cursos con el título Les 
de obras de Husserl. Imparte estos cursos - nada menos que cuatro cursos de duración anual cada uno de ellos - en la Sorbona de 1933 a 1937, hasta que, de nuevo, se ve obligado al exilio. Esta vez se exilia a los Estados Unidos, en donde, después de unos años sin poder asentarse profesionalmente en su especialidad, en 1959 lo invitan a ser profesor de filosofia en la Graduate Faculty of Political and Social Science, en la New School for Social Research, en la ciudad de New York, por entonces centro máximo de la fenomenología estadounidense. Ocupará ahí la plaza que había dejado vacante la muerte de Alfred Schütz. Y a raíz de esta etapa en la New School hay una gran coincidencia entre los que se refieren a los inicios del pensamiento fenomenológico en ese país: dicen de Aron Gurwitsch que él, es, sin duda, "la gran figura de la fenomenología norteamericana". 5 Como consecuencia de haber pasado unos treinta y seis años en los Estados Unidos, hasta su fallecimiento en 1973, la mayor parte de su producción está publicada allí y redactada en inglés. Lamentablemente esto, que ha sido muy afortunado para la fenomenología de ese país americano, tiene una contrapartida negativa: ha ocasionado un gran desconocimiento en la Europa continental de la obra de Aron Gurwitsch y de su valiosa aportación.

Consciente, pues, de este desconocimiento y convencida de que ya es hora de superarlo por parte de nuestra filosofía europea reconociendo la contribución fenomenológica de Aron Gurwitsch, con esta ponencia pretendo contribuir de algún modo a animar al redescubrimiento de este gran fenomenólogo con el que todos tenemos una deuda y sin el cual, ciertamente, Merleau-Ponty nunca habría sido el Merleau-Ponty que todos conocemos, como he mostrado en otro lugar. ${ }^{6}$ Trazar la historia de nuestra tradición fenomenoló-

tendences actuelles de la Philosophie alemande: E. Husserl, M. Scheler, E. Lask, N. Hartmann (Paris, Vrin, 1930). Él mismo dice que el curso sobre Husserl contiene aquí algunas ampliaciones. Pero, con todo, este texto escrito y, por tanto, la conferencia que le ha servido de base, no tiene mucha extensión. Y si Georges Gurvitch consigue atraer el interés de ese público francés hacia Husserl posiblemente es debido, en buena medida, a su acierto en hacer una exposición de la filosofia de Husserl que, en algunos aspectos, se veía muy próxima a la filosofia de Henri Bergson, en esos momentos figura de enorme peso intelectual en Francia.

5 Es éste el sentir generalizado en torno a él, y así lo expresa haciendo de portavoz de este sentir, por ejemplo, su discípulo Lester Embree. Cfr. su Introducción a la traducción castellana de "On Contemporary Nihilism" en Agora. Papeles de Filosofia vol. 22, nº 2 (2003), p. 161.

6 Cfr. "Gurwitsch, Goldstein, Merleau-Ponty. Analyse d'une étroite relation", en R. Barbaras / M. Carbone / L. Lawlor (eds.) Chiasmi International: Merleau-Ponty. Entre Esthétique et Psychoanalyse, Paris / Milano / Memphis / Manchester, Vrin / Mimesis / University of Memphis / Clinamen Press, 2004, pp. 147-171.

El libro de Kurt Goldstein Der Aufbau des Organismus ha sido traducido al francés y publicado en 1951 por la editorial Gallimard con el título La structure de l'organisme. Hiere mi sensibilidad intelectual uno de los párrafos del "Préface" de Pierre Fédida. Con sus palabras, demuestra una vez más que siempre se ha pasado por alto quien ha introducido y divulgado las investigaciones de Goldstein en la filosofia francesa. Fedida afirma que "c'est certainement à Maurice Merleau-Ponty que nous devons, pour l'essentiel, la frécuentation 
gica europea requiere poner a Aron Gurwitsch en el puesto que le corresponde. Esto nos conduce directamente a recuperarlo por su continuado trabajo en fenomenología de la percepción desde la segunda mitad de los años veinte. Porque, en efecto, como intentaré apuntar en lo que sigue, así es como él entiende la fenomenología: una fenomenología de la percepción de la que dan buena fe tanto los ensayos de su etapa alemana, como los de su etapa francesa como los de su posterior etapa estadounidense. Y si estos ensayos dan fe de ello, las siguientes palabras del propio Gurwitsch recogen de primera mano esta intención y este trabajo. Estas palabras suyas pertenecen a un "esbozo de un proyecto de libro para ser titulado Fenomenología de la percepción". Dice así:

El libro proyectado tiene la finalidad de suministrar una teoría fenomenológica de la percepción basándose en los logros alcanzados por Husserl y por su escuela, pero yendo en la medida de lo posible más allá de estos logros...Los primeros estudios [para este libro] comenzaron en 1938/1939. En la primavera de 1939 el solicitante dio clases en Paris sobre algunos temas relaconados [con este libro en proyecto] y desde ese momento ha continuado recogiendo material y desarrollando los puntos de vista. En el verano de 1939, la Caisse Rationale de la Recherche Scientifique en París nombró al solicitante becario investigador para permitirle que continuase con los estudios preparatorios y que escribiese el libro. El estallido de la guerra interrumpió la investigación. Después de su llegada a este país [esto lo está escribiendo ya en Estados Unidos] -en mayo de 1940-, el solicitante retomó sus estudios sobre el libro proyectado.

des travaux de Kurt Goldstein... " (p. X). Si Merleau-Ponty no hubiera trabajado en sus años de investigación doctoral la obra de Gurwitsch publicada en 1929 (Phänomenologie der Thematik und des reinen Ich. Studien über Beziehungen von Gestalttheorie und Phänomenologie), si no hubiera conocido personalmente a Gurwitsch en 1934, si no hubiera asistido a los cuatro cursos anuales impartidos por Gurwitsch en la Universidad de la Sorbona, si no hubiera tenido la oportunidad de tener continuas charlas con Gurwitsch sobre toda la temática en la que trabajaba Gurwitsch y que coincidía con los propios intereses merleaupontyanos, etc., entonces, Fedida podría tener razón en lo que dice. Pero las cosas se desarrollaron de otra manera. Y sólo es necesario estar muy atento a la cronología de los hechos y documentos para concederle a cada uno lo suyo. De ahí que mi ensayo citado arriba en esta nota finalice planteando esta cuestión retórica: "Sans Gurwitsch, Merleau-Ponty aurait-il écrit ses deux Thèses et tenté d'annexer Goldstein et Husserl?" (p. 165). Las dos Tesis son La structure du comportement y Phénoménologie de la perception. En ellas, desconcertantemente, Merleau-Ponty no reconoce en ningún momento su deuda con su maestro Aron Gurwitsch. Y, por consiguiente, él es el que inicia la confusión que hasta el momento hemos estado arrastrando.

No hay para mí ninguna duda de que fue Gurwitsch quien introdujo a Merleau-Ponty en Goldstein. Porque, aunque el enfoque de Goldstein ya aparece recogido por Ernst Cassirer en su tercer tomo de Filosofia de las formas simbólicas, y que esta obra es de 1923-29, sin embargo, no hay en él un tratamiento expositivo completo del pensamiento goldsteniano. 
Mientras tanto ha publicado algunos artículos más breves o más o menos relacionados con el libro proyectado. ${ }^{7}$

Lester Embree, al recuperar y editar el esbozo de este libro y el texto al que pertenecen estas palabras, explica que:

esta propuesta para una beca probablemente remitida a la Fundación Ford en 1943 fue encontrada entre los papeles de Aron Gurwitsch después de su muerte. Documenta la situación intelectual a la que se enfrentó en ese momento, el intento (asimismo como el título original) de su obra magna EL CAMPO DE LA CONCIENCIA, y, sobre todo, su proyecto filosófico de continuar de forma crítica la fenomenología constitutiva inaugurada por Edmund Husserl. ${ }^{8}$

Aron Gurwitsch, como fiel discípulo de Edmund Husserl, hace suyas la actitud, la metodología y las principales convicciones husserlianas. ${ }^{9} \mathrm{Sin}$ embargo, aun siendo así, en sus exposiciones y análisis de la fenomenología tiene su propio estilo, y éste consiste en centrarse más en unos aspectos que en otros sin por ello descuidar el conjunto. Es este estilo suyo el que yo aquí intentaré exponer mostrando fundamentalmente dos cosas: la primera, que Gurwitsch entiende la fenomenología como "fenomenología de la percepción" (lo cual no podría ser de otro modo, ya que la fenomenología husserliana o es fenomenología de la percepción o no es fenomenología) y que es así como logra transmitirla en sus cursos docentes y en sus escritos, y, la segunda, que los aspectos de esta fenomenología en los que más focaliza su atención desde el principio le conducen a sentir una profunda sintonía con el trabajo del neu-. ropsiquiatra Kurt Goldstein. Hay, por tanto, dos líneas de investigación que se juntan en este pensador interesado en la percepción desde los inicios de su andadura filosófica: por una parte está su contacto directo con el Husserl de Friburgo y su profunda compenetración con él; por otra parte está su trabajo en Frankfurt con el neuropsiquiatra Goldstein y con el psicólogo con el que

7 "Outline of a projected book to be entitled Phenomenology of Perception". Este texto está recogido en Lester Embree (ed.) Essays in Memory of Aron Gurwitsch, Washington D.C., Center for Advanced Research in Phenomenology / University Press of America, 1984, pp. $8 \mathrm{~s}$.

8 Ibid., p. 3.

9 Lester Embree hace referencia a valiosos datos de la biografía de Gurwitsch. Entre ellos, por ejemplo, los siguientes: En la década de los veinte, estudió filosofía y psicología con Stumpf, en Berlin. Stumpf le recomienda ir a Husserl. Gurwitsch asiste al curso que Husserl imparte en Friburgo sobre Natur und Geist. Se va a Frankfurt a trabajar con Goldstein y con Gelb sobre los efectos psicológicos causados por las lesiones cerebrales en heridos de la primera Guerra Mundial. En diciembre de 1929 Husserl comienza a leer una copia de la Tesis Doctoral de Gurwitsch que éste le ha enviado. Y, a partir de entonces, Gurwitsch visita a Husserl en Friburgo en varias ocasiones y en una relación que mantendrán hasta la muerte de Husserl. Cfr. su Préface a Esquisse de la phénoménologie constitutive, Paris, Vrin, 2002. En adelante, este texto será citado como Esquisse. 
Goldstein trabajaba: el gestaltista Adhémar Gelb. Muy pronto se dio cuenta Gurwitsch de que era mucho lo que tenían en común los enfoques de Husserl y los de Goldstein y la psicología de la Gestalt -a la que Goldstein estaba tan próximo sin pertenecer a ella. ${ }^{10}$ Mostró y defendió esta convicción en su Tesis Doctoral de 1928, la cual publicó inmediatamente, en 1929, con el mismo título: Phänomenologie der Thematik und des reinen Ich. Studien über Beziehungen von Gestalttheorie und Phänomenologie. ${ }^{11}$ Él mismo dirá de este trabajo de investigación que buscaba ideas de la psicología gestaltista que son fecundas para la fenomenología y que, de este modo, deseaba hacer avanzar la fenomenología llevándola, si acaso, incluso más allá de Husserl. ${ }^{12}$

Su relación con Goldstein comenzó en el Instituto de Frankfurt en 1921 $y$, desde entonces, su trabajo con él y con Gelb marcó el enfoque de toda su investigación fenomenológica. Además de su Tesis Doctoral, de 1928, y (parcialmente) de su Tesis de Habilitación, de 1931 (Die mitmenschlichen Begegnungen in der Milieuwelt) ${ }^{13}$ dedicó cuatro escritos íntegros a la teoría goldsteniana,${ }^{14}$ el tercero de los cursos que impartió en la Sorbona (en 1935-36) es-

10 Debemos aclarar que en ese momento es muy probable que Gurwitsch considerase que Goldstein se movía dentro de la Escuela de la Gestalt. Sin embargo, el propio Goldstein aclarará unos años después, en 1934, que "notre conception centrale coïncide en bien des points avec la psychologie de la forme. Toutefois, elle ne proviene pas de celle-ci et n'est pas, comme on l'a prétendu, une application. Ce n'est pas pour des raisons de priorité que je mentionne ce fait -je reconnais ouvertement et volontiers que cette doctrine et ses représentants m'ont appris bien des choses que j'ai utilisées, et dans ma conception fondamentale et dans le détail- mais cette façon de voir laisse facilement inaperçues les diferences que existen entre la psychologie de la forme et ma théorie". Cfr. La estructure de l'organisme, Paris, Gallimard, 1983, p. 405 [319].

11 Phänomenologie der Thematik und des reinen Ich. Studien über Beziehungen von Gestalttheorie und Phänomenologie, en Psychologische Forschung, Bd. 12. Hay traducción inglesa: Phenomenology of Thematics and of the Pure Ego: Studies of the Relation between Gestalt Theory and Phenomenology, en Aron Gurwitsch Studies in Phenomenology and Psychology, Evanston, Northwestern University Press, 1966.

12 Dice Gurwitsch que en Phänomenologie der Thematik und des reinen Ich "nous avons cherché là à rendre fécondes quelques vues de la théorie de la forme pour l'analyse phénoménologique des faits en question, et nous avons essayé de la faire avancer ainsi au-delà de l'état dans lequel Husserl l'a laisée, tout en lui restant redevable non seulement de quantité de suggestions, mais avant tout de la direction dans laquelle nous avons cherché a faire progresser les recherches". Esquisse, p. 142

13 Berlin/New York, Walter de Gruyter, 1977. Alexandre Metraux ha editado la traducción inglesa: Human Encounters in the Social Wordl, Pittsburg, Duquesne University Press, 1979.

14 "Le fonctionnement de l'organisme d'après M. K. Goldstein", Journal de Psychologie Normale et Pathologique XXXVI (1939) 107-138. "La science biologique d'après M. K.Goldstein", Revue Philosophique de la France et de l'Étranger CXXIX (1940) 244-265. "Gelb-Goldstein's Concept of 'Concrete' and 'Categorial' Attitude and the Phenomenology of Ideation", Philosophy and Phenomenology Research 10 (1949) 172-196. "Einleitung” a Kurt Goldstein. Selected Papers / Ausgewählte Schriften, The Hague, M. Nijhoff, 1971. El segundo texto se ha reeditado de nuevo tres veces: (1) "Goldstein's Conception of Biological Science", en Studies in Phenomenology and Psychology, pp. 69-88; (2) "Goldstein's 
taba consagrado a la "actitud categorial" de Goldstein, y, en general, en toda su obra hay frecuentes citas de Goldstein. Y, al igual que antes decíamos de Gurwitsch con respecto a la introducción que hace en Francia de la fenomenología husserliana lo mismo tenemos que decir ahora con respecto a la introducción que hace en Francia de Goldstein, ya que antes de 1935-36, es decir, antes de que imparta su curso sobre él, no se había escrito allí nada acerca de Goldstein $^{15}$. En mi opinión, es destacable el hecho de que Gurwitsch, al final de su vida, continúa pensando de este neuropsiquiatra exactamente lo mismo que pensaba cuando trabaja en él para su Tesis Doctoral y de que haya mantenido un diálogo con él durante toda su vida; ambos hechos demuestran que el interés de Gurwitsch por Goldstein ni mucho menos se limitaba a lo que él le podía aportar para la elaboración de su Tesis Doctoral. Cuando en 1971 (dos años antes de su fallecimiento) hace la Introducción de un libro recopilación de diversos escritos de Goldstein, Gurwitsch sigue diciendo de las interpretaciones golsdstenianas lo mismo que ya decía en su primer trabajo de investigación de 1928: que éstas "están llamadas a efectuar una muy importante contribución para el impulso de ciertos problemas de la fenomenología de Husserl. ${ }^{16}$ Durante toda su vida, Gurwitsch permaneció en contacto con Goldstein, en una relación de fuerte amistad; incluso cuando los dos -ambos judíos exiliados- estaban residiendo en Estados Unidos. ${ }^{17} \mathrm{Y}$ como dato muy significa-

Conception of Biological Science, en. J. Kockelmans (ed.) Phenomenology, New York, Doubleday Anchor, 1967, pp. 118-136.; (3) "La science biologique d'après M. K. Goldstein", en Esquisse de la phénoménologie constitutive, Paris, Vrin, 2002, pp. 369-387. El tercer texto está reeditado en Studies in Phenomenology and Psychology, pp. 359-384. En general, para los datos de todos los ensayos de Aron Gurwitsch, cfr. su website en http://www.phenomenologycenter.org $\rightarrow$ http://www.gurwitsch.net.

15 Marc Klein escribió un extenso artículo en 1954 con motivo de la publicación, en 1951, de la traducción francesa de Der Aufbau des Organismus. En este artículo menciona todos los escritos que hasta entonces se publicaron en Francia sobre el pensamiento de Goldstein. Si atendemos a los datos que aquí nos brinda, el caso es que mientras este curso de Gurwitsch sobre Goldstein lo imparte en 1935-36, el primer texto referido a Goldstein por parte de otros autores es de 1937, de R. Mourgue, publicado en una revista de neurología. Los siguientes escritos sobre Goldstein son de 1938, de 1942, de 1943, y de ahí en adelante. Cfr. Marc Klein "Sur les résonances de la philosophie de la nature en biologie moderne et contemporaine", Revue Philosophique de la France et de l'Étranger CXLIV (1954), pp. 515.

16 "Einleitung" a Kurt Goldstein. Selected Papers / Ausgewählte Schriften, The Hague, M. Nijhoff, 1971, p. XI.

17 De ello da fe una carta que L. Van Breda le escribe a Gurwitsch el 15 de junio de 1954, después de la estancia de Van Breda en la Universidad estadounidense de Brandeis a donde acudió para disertar sobre Husserl. En esta carta, Van breda le agradece a Gurwitsch el haberle facilitado la dirección del profesor Goldstein y el haber tenido la gentileza de incluso hablarle a Goldstein de este su admirador en Lovaina. Se disculpa de no poder precisar en ese momento la fecha de su próxima visita a Estados Unidos y le pide a Gurwitsch que él mismo le haga el favor de explicarle a Goldstein las razones que en esta carta aduce para no poder precisar todavía esa fecha. 
tivo valga también el de que encima de su mesa de trabajo tenía un retrato de Goldstein, su querido maestro y amigo (al igual que una foto de Husserl presidía la pared que hacía de fondo de la mesa).

\section{II}

Por tanto, si esto es así, la pregunta de base que actuará para mí como guión para elaborar esta ponencia es qué ve Gurwitsch en el análisis que hace Goldstein de sus pacientes con lesiones cerebrales para considerar él que este análisis goldsteniano es extremadamente coincidente con el propio análisis fenomenológico de la percepción de Husserl.

Sin duda, para Gurwitsch, hay una primera coincidencia básica entre estos dos autores en su posicionamiento ante la psicología de la época - la cual coincidencia es básica tanto porque esto en lo que coinciden está en el punto de partida de cada uno por separado como porque esto es la pieza que hace de base sobre la que se sostiene el pensamiento de cada uno de ellos. En efecto, cuando Gurwitsch habla de la posición de Husserl ante la psicología de su época ${ }^{18}$ y cuando habla de la posición de Goldstein ante la psicología de su época, ${ }^{19}$ expone posturas extremadamente coincidentes entre ellos; coincidentes en el doble sentido de que, en primer lugar, ambos parten de una crítica de esta psicología y, en segundo lugar, de que ambos ven la necesidad de elaborar un nuevo concepto de conciencia y, por tanto, de un nuevo enfoque de la psicología. En efecto, la psicología de esa época tenía en su base la dualidad cartesiana. El sujeto está compuesto de dos sustancias que permanecen separadas

Esta carta está depositada en el archivo del Center for Advanced Research in Phenomenology (CARP) de la Florida Atlantic University. Debo agradecerle al discípulo de Aron Gurwitsch, Lester Embree que, como presidente del CARP, amablemente me haya permitido consultar los documentos de Gurwitsch contenidos en este archivo, al igual que le agradezco las largas horas de conversación que hemos mantenido -y que seguimos ahora manteniendo por correo electrónico y en nuestros anuales encuentros junto al mar de Galicia- sobre diversos aspectos de la vida y obra de Aron Gurwitsch. Yo he descubierto la importancia de Gurwitsch dentro de la fenomenología gracias, y sólo gracias, a la razonada y entusiasta insistencia de Lester Embree y con él también he aprendido a quererlo y a reconocer su gran valía y su enorme peso en la orientación del trabajo fenomenológico de Merleau-Ponty. Por tanto, mis intentos no son más que un deseo de continuar el afán embreeano de recuperar a Gurwitsch en ese determinante papel que en su momento jugó dentro marco de la fenomenología europea.

18 Cfr. Aron Gurwitsch "The Last Work of Edmund Husserl" (second part), Philosophy and Phenomenological Research (Buffalo, New York), vol. XVII (1957). Hay traducción en castellano: "La última obra de Edmundo Husserl" (segunda parte), Lecciones y Ensayos (Buenos Aires) n 7 (1958) 7-33.

19 Cfr. Esquisse, cap. I, §4. Dice aquí Gurwitsch que, según Goldstein, "il faut abandonner l'idée que les faits physiologiques et les faits psychiques constitueraient deux plans indépendants et séparés par leur nature. L'auteur va même jusqu'à dénoncer comme mal posé le problème de l'interdépendance et de $\mathrm{l}^{\prime}$ interaction psycho-physiques." 
y que, siendo heterogéneas entre sí, dan lugar a un sujeto escindido en dos planos que son independientes por su propia naturaleza: hechos psíquicos (lo psíquico) y hechos físiológicos (lo físico). La psicología de ese período de entre siglos XIX-XX se estaba ocupando de la experiencia "interna" para analizarla cartesianamente como si se tratara de un hecho físico, de una realidad (res) más, tal como hace la física cuando estudia la realidad "externa". Nace así la psicofísica, la cual se ve a sí misma como una rama más de la biología al entender la conciencia como formada por hechos fisiológicos. Pues bien, la exposición que Gurwitsch hace de Husserl y de Goldstein muestra a ambos autores, en su punto de arranque, coincidiendo en su disconformidad con esta concepción dualista del sujeto y con el consiguiente enfoque y metodología de esta psicología, una psicología que nunca elaboró su concepto de lo psicológico sobre la base de la experiencia directa sino que se limitó a adoptar esa concepción dualista cartesiana, la cual, a su vez, había sido determinada por las ideas de una naturaleza meramente corporal y de una ciencia matemática de esa naturaleza corporal. Hay, pues, que elaborar una nueva concepción de la conciencia, dirá Husserl; hay, pues, que elaborar una nueva concepción del organismo, dirá Goldstein ${ }^{20}$ (obviamente, ambos se están refiriendo a la necesidad de cambiar el concepto de sujeto manejado hasta entonces). Para ello, la psicología tendría que partir, para Husserl, de los datos del mundo vivido (Lebenswelt), y respetarlos. Y esto le llevaría a cómo cada individuo, en cuanto sujeto de este mundo, experimenta el hecho de habitar y de funcionar (walten) en nuestro cuerpo - experiencias que en su forma primordial y originaria sólo se pueden tener con respecto al propio cuerpo de cada cual. En otras palabras: debemos partir de los seres humanos como existentes que se ocupan, de modos diversos, de objetos, hechos, cosas, etc., dentro de su mundo de la vida. Lo cual significa que debemos partir de la conciencia perceptiva como intencional. Conciencia y mundo forman una inextricable relación intencional, una unidad intencional; en ella, el objeto ("objeto intencional") es lo que es para el sujeto según se le presenta en su percepción y según es vivido por él. Y el psicólogo lo que debe, pues, hacer, según Husserl, es ponerse en cómo se le aparece la realidad al sujeto y en cómo ésta es experimentada por él, sin juzgarla ni ponerla en tela de juicio. Por ejemplo, al estudiar un acto de percepción, el psicólogo deberá atenerse escrupulosamente a la cosa percibida tal como es percibida efectivamente, no debiendo pararse en si la cosa percibida existe en la realidad o de si es tal como se la percibe. El psicólogo (es decir, el psicólogo que ha efectuado un cambio de actitud -el de la reducción fenomenológico-psicológica (por tanto, el fenomenólogo)- se ocupará tan sólo de "objetos intencionales". ${ }^{21} \mathrm{Y}$, de este modo, la fenomenología husserliana está

${ }^{20}$ Como médico, como neuropsiquiatra, Goldstein va al sujeto desde la perspectiva de la biología. De ahí que hable de "organismo", es decir, del organismo del sujeto viviente, rechazando que éste pueda ser reducido a una colección de hechos fisiológicos. 
invitando a ir para atrás y a alcanzar ese punto de la experiencia perceptiva en donde todavía no hay escisión cartesiana, en donde todavía nos hallamos ante la unidad primitiva. Porque lo "psiquico" y lo "físico" no son realidades dadas, sino tan sólo el resultado de una separación abstracta que lo que hace es modificar -analíticamente, abstractamente- la realidad vivida y concreta en la que está el sujeto perceptivo ${ }^{22}$. En términos fenomenológicos, sujeto y mundo (mundo vivido) forman, pues, una misma unidad intencional originaria.

Gurwitsch se dio cuenta ya en su etapa de investigación doctoral de que los trabajos de Kurt Goldstein se apoyan en una postura muy semejante a ésta de Husserl. Goldstein estaba dedicado a describir y analizar el tipo de relación perceptiva y de comportamiento práxico que los pacientes tienen con su mundo (cómo se relacionan con objetos, con hechos, con otras personas, etc.). $\mathrm{Y}$, cuando así procede, de una u otra manera lo que está haciendo es describir $\mathrm{y}$ analizar la intencionalidad de la conciencia de sus pacientes, para lo cual antes, como neuropsiquiatra, ha tenido que efectuar un cambio de actitud (epojé), poniendo en suspenso las diversas teorías explicativas habidas hasta ese momento, y un querer re(con)ducir a lo que es vivido y experimentado por sus pacientes (reducción fenomenológica) cuando ellos están percibiendo y ejecutando alguna actividad. Para él, uno debe ceñirse a captar y a describir, ateniéndose a los hechos dados en observación y renunciando provisionalmente a toda interpretación de ellos. ${ }^{23}$ Los hallazgos a los que da lugar el trabajo de Goldstein enfocado de este modo - un trabajo llevado a cabo de forma completamente independiente al de Husserl - permitirán confirmar, según Gurwitsch, hallazgos fundamentales de la fenomenología husserliana. Esto es lo que a continuación veremos en distintos pasos.

Para Goldstein, el organismo (es decir, lo que para nosotros es el "sujeto") es un todo organizado y nunca una suma de partes independientes; cada "parte" está en dependencia funcional recíproca con cada una de las otras. Así, el organismo no es más que el conjunto articulado y centralizado de sus "partes", y siempre es todo el organismo el que está comprometido en toda acción, en toda reacción, incluso si éstas son efectuadas sólo por algún órgano. ${ }^{24}$ Esto

22 Cfr. Esquisse, cap. I, $\S 4$, pp. 67s.

23

Cfr. "La science biologique d'après M. K. Goldstein", p. 392. Dice en su Tesis Doctoral que, "entendido de este modo el procedimiento de la Gestalttheorie, al tomar en cuenta lo psíquico puramente de modo descriptivo dejando de lado todas las construcciones, tiene la misma significación y función metodológica para la psicología como la reducción trascendental tiene para la fenomenología". Cfr. La versión inglesa Phenomenology of Thematics and of the Pure Ego: Studies of the Relation between Gestalt Theory and Phenomenology, en Aron Gurwitsch Studies in Phenomenology and Psychology, Evanston, Northwestern University Press, 1966, p. 194.

24 Cfr. Esquisse, cap. I, § 4,, p. 69 y “La science biologique d'après M. K. Goldstein”, p. 384. El texto de Goldstein en el que más se basa Gurwitsch en su exposición de él es "L'analyse de l'aphasie et l'étude de l'essence du langage", publicado en Journal de Psychologie Normale et Pathologique 30 (1933) 430-496, y reeditado en Aron Gurwitsch / Else M. Goldstein Hau- 
es lo que significa en Goldstein el concepto de la estructura del organismo recogido en su libro de 1934 Des Aufbau des Organismus. Para Goldstein dice Gurwitsch -, "únicamente a partir de la estructura esencial de un organismo es posible apreciar la importancia y la significación de lo somático y, sobre todo, de lo psíquico para la vida de este organismo". ${ }^{25}$ Por tanto, "la consideración biológica debe partir no de la separación arbitraria y fáctica de lo "físico" y de lo "psíquico", sino de esta unión estrecha e íntima en la que se encuentran en el organismo viviente". ${ }^{26}$ Para Goldstein, pues, al igual que antes para Husserl, lo psíquico y lo somático forman una unidad originaria que es necesario recuperar. Pensemos que en esa época, algunos autores tomaban los fenómenos somáticos como primarios y los hechos psíquicos como dependientes de estos, mientras que otros tendían a ver los hechos somáticos como condicionados por los psíquicos. El caso es que, para todos ellos por igual, esa acción de unos hechos sobre otros, ya sea concebida como unilateral o ya como recíproca, solamente puede ser admitida como tal después de haber tomado lo psíquico y lo somático como dos planos no sólo diferentes sino incluso separados entre sí. Y esto es para Goldstein lo que constituye - señala Gurwitsch - un error mayúsculo. ¡No se puede romper la unidad originaria, la unidad del organismo! Al contrario, es necesario partir de esta unidad y a ella remitir los actos que abstractamente "designamos" como somáticos o como psíquicos, puesto que estos actos forman una unidad y no deben ser considerados más que como manifestaciones vitales de esta unidad, sin primacía de los unos sobre los otros, por mucho que los actos somáticos y los psíquicos tengan cada uno su naturaleza particular. ${ }^{27}$ Esta concepción goldsteniana significa también que los fenómenos que acontecen en el organismo deben ser explicados relacionándolos con la situación concreta en donde se encuentra dicho organismo en cuestión. ${ }^{28}$ En realidad, para Goldstein, al igual que para Husserl, el medio (Umwelt) no preexiste al organismo ni se le impone desde fuera. El medio no dirige al organismo sino que es éste el que selecciona y decide entre los hechos exteriores y, así, responde a ellos sin tener en cuenta todo lo demás de ese medio de lo que en cada momento prescinde. El organismo es él y su situación vital en un medio entorno, es decir, él y las tareas que conlleva para él el estar en una situación vital de conjunto. ${ }^{29}$ Podemos expresar esto en

dek / Willian E. Haudek (eds.) Kurt Goldstein. Selected Papers / Ausgewählte Schriften, The Hague, Nijhoff, 1971, pp. 282-344.

25 "La science biologique d'après M. K. Goldstein", p. 384.

${ }^{26}$ Esquisse, cap. I, § 4, p. 69.

27 Cfr. "La science biologique d'après M. K. Goldstein", en Esquisse, pp. 383s.

28 "Notre troisième exigence méthodologique est de ne considérer aucun phénomène sans référence à l'organisme et à la situation dans laquelle on l'observe". K. Goldstein La structure de l'organisme, Paris, Gallimard, 1951, pp. 23s.

29 "Le fonctionnement de l'organisme d'après M. K. Goldstein", p. 112-114. Goldstein pertenece a ese grupo de científicos que inauguran, cada uno, desde su parcela de saber, una Nueva Ciencia con el nuevo cambio de paradigma que esto trae consigo. Esto sucede en el 
los mismos términos husserlianos de los que antes echamos mano: el sujeto y su mundo forman una misma unidad intencional. Esto es: para Goldstein, al igual que para Husserl, siempre hay que analizar a un organismo tomándolo en esa situación vital en la que está inmerso, porque sólo contemplándolo así podremos apreciar el papel jugado ya por lo psíquico ya por lo físico en esas condiciones concretas. En otras palabras, ambos autores están proponiendo abandonar los esquemas objetivistas decimonónicos y analizar cómo vive el sujeto su situación vital, cómo la experimenta, cuál es la experiencia perceptiva que de ella tiene y que él, y sólo él, está viviendo desde sí mismo.

Es precisamente éste el enfoque con el que Goldstein aborda sus investigaciones con sus pacientes. Su propósito es describir y analizar cómo ha cambiado la actitud de estos pacientes hacia el mundo exterior (que a ellos llega perceptivamente), cómo el mundo (perceptivo) les afecta ahora de otro modo y cuál es el nuevo sentido que pasan a tener las cosas para ellos. Y lo que Goldstein nos proporciona como fruto de sus observaciones clínicas es que para sus pacientes con lesiones cerebrales las cosas tienen un sentido estrictamente concreto ${ }^{30} \mathrm{El}$ marco teórico goldsteniano de esta afirmación es la diferenciación que él establece entre "actitud concreta" y "actitud categorial". 31

Gurwitsch ve en esta diferenciación de actitudes una simetría con la diferenciación que hace Husserl entre lo categorial y lo que se da inmediatamente en la experiencia sensorial sin que intervenga ningún elemento exterior a esta experiencia sensorial (es decir, ningún pensamiento categorial). Husserl descubre que términos tales como la "identidad", la "semejanza", la "unidad", etc., tienen una doble significación. Por ejemplo, "la cuestión de saber como se puede discernir de una sola mirada el carácter de unidad de una multiplicidad cualquiera sin tener ni la necesidad ni el tiempo de percorrer, uno a uno, todos los miembros que la componen, ha conducido a Husserl - dice Gurwitsch - a establecer una separación entre la unidad pensada, categorial, artificial, por tanto, y la unidad inmediatamente sentida, natural...(en la) que no

período de entre siglos XIX-XX. Por ejemplo, Ferdinard Saussure inaugura la Nueva Lingüística, Henri Berr la Nueva Historia, los gestaltistas Wertheimer, Köffka y Köhler la Nueva Psicología, Vidal de la Blanche la Nueva Geografía, Marcel Mauss la Nueva Antropología Social, Jacob Von Uexküll la Nueva Biología, K. Goldstein la Nueva Neuropsiquiatría y E. Husserl la Nueva Filosofía. En general, todos estos autores mencionados comparten un mismo rechazo por los decimonónicos esquemas "objetivistas", tienden a recuperar la subjetividad y buscan lo concreto y lo "vivido". Es interesante notar que la consideración goldsteniana de que, biológicamente hablando, sujeto y mundo forman una unidad y de que el sujeto no es un mero receptor que reacciona pasivamente ante el medio, es en extremo semejante a la consideración de Von Uexküll en Biología. Y entre Von Uexküll y Husserl hay una gran concordancia. Cfr. María Luz Pintos "La fenomenología y las ciencias humanas y bio-sociales. Su convergencia en un importante momento de cambio de paradigmas", Revista Philosophica (Valparaíso, Chile) nº 27 (2004) 215-245.

${ }^{30}$ Cfr. K. Goldstein "L'analyse de l'aphasie et l'étude de l'essence du langage”, p. 332.

31 Ibid., pp. 325ss. 
interviene ningún pensamiento categorial". ${ }^{32}$ Con respecto a la unidad y los demás términos categoriales, Husserl considera que pueden darse en sentido conceptual y también en un sentido dado de modo inmediato por ser inmanentes a las propias experiencias de percepción sensoriales. ${ }^{33}$ Esta diferenciación es fundamental y muy importante. Husserl cree que, por ejemplo, cuando yo entro en una sala y veo (lo que para mí es) un "conjunto" de personas, este carácter de unidad de ese "conjunto" es inherente a la propia percepción que estoy efectuando, es un hecho inmediato, sin que tenga que ser constituido categorialmente, es decir, pensado de modo racional. Para Husserl -dice Gurwitsch- el carácter de unidad, de grupo, es un hecho "inmediatamente percibido" porque en el momento mismo de la percepción sensorial se produce una fusión entre los elementos y entre las relaciones de estos elementos que hace que se constituyan en un grupo cuya unidad es perceptiva e inmediata y no depende de nada exterior a la misma percepción, es decir, de ninguna actividad mental por encima de la actividad perceptiva en sí misma. Se trata del "factor figural". ${ }^{34} \mathrm{Su}$ reconocimiento por parte de Husserl entraña unas consecuencias de trascendental alcance para su fenomenología y es indudable que, como veremos, estamos en un punto de coincidencia clave entre Husserl y Goldstein según la lectura que de ellos hace Gurwitsch.

Permítanme acudir ahora a un ensayo de Gurwitsch titulado "Présuppositions philosophiques de la Lógique". ${ }^{35}$ En este ensayo, tanto por lo que en él explica sobre Husserl como por los textos de Husserl que ahí toma de referencia para apoyar su explicación (el título de este ensayo delata por sí mismo que se apoya fundamentalmente en Formale und Transzendentale Logik y Erfahrung und Urteil), se evidencia lo que a Gurwitsch le ha impactado tan fuertemente de la fenomenología husserliana y lo que, a su vez, él ha intentado trasmitir siempre en sus clases y en su obra escrita: para él, el análisis regresivo husserliano conduce directamente a la experiencia perceptiva anterior a toda operación de pensamiento categorial, es decir, a la experiencia ante-predicativa. Es esto - dice - lo que Husserl pretende estudiar como la génesis del conocimiento porque sólo la toma de conciencia de la experiencia ante-predicativa nos permitirá captar el surgimiento de evidencias de otros grados; de ahí que debamos hacer una "regresión" y llegar a los sustratos últimos de todo nuestro pensamiento. De acuerdo con esto, dice Gurwitsch, en el enunciado del programa de Husserl "la primera tarea que se traza es dar cuenta, en

32 A. Gurwitsch "Quelques aspects et developpements de la Psychologie de la Forme", Journal de Psychologie Normal et Pathologique 33 (1936) p. 316.

33

Cfr. A. Gurwitsch "Einleitung" a Kurt Goldstein. Selected Papers / Ausgewählte Schriften, pp. XXIIss.

34 Cfr. A. Gurwitsch "Quelques aspects et developpements de la Psychologie de la Forme", y Cfr. también Esquisse en su capítulo IV, § 2.

35 Este texto que originalmente se publicó en el n ${ }^{\circ} 4$ de la Revue de Métaphysique et de Morale (1951) se volvió a publicar en H. L. Van Breda (ed.) Phénoménologie. Existence, Paris, Vrin, 1954. Aquí citaré por esta segunda publicación. 
términos de conciencia constituyente, del mundo perceptivo en el que [ya] nos encontramos y en el seno del cual se desenvuelven nuestras vidas y todas nuestras actividades, mundo que nos es familiar en nuestra experiencia de todos los días". ${ }^{36}$ Porque incluso los conceptos categoriales que manejamos en nuestra vida cotidiana e, incluso, los que se usan en las ciencias lógico-matemáticas, todos tienen, según esto, sus raíces en la experiencia perceptiva, en la experiencia perceptiva del mundo en el que vivimos y que nos es dado presupuesto en todas nuestras actividades de todo tipo.

Parece, pues, que su profunda penetración en la actitud fenomenológica le había permitido a Gurwitsch hacer muy suya esta principal idea husserliana; idea que pasará a ser, también, una de sus grandes convicciones y que, al igual que en Husserl, atravesará toda su obra investigadora y docente de un lado a otro. Gurwitsch expresa así, concisamente, esta idea husserliana: "El sentido último del mundo no puede ser obtenido más que del análisis de la vida perceptiva". ${ }^{37} \mathrm{Y}$ el mundo perceptivo es el mundo tal como figura en la vida natural y cotidiana de todos los días. "El mundo perceptivo es el mundo en el que nos encontramos, es decir, en el que actuamos, reaccionamos y trabajamos. Es en este mundo en donde nosotros reconocemos a nuestros semejantes a los que estamos ligados por las relaciones más diversas...Toda nuestra vida afectiva y. emocional se refiere a este mundo; y es, de nuevo, a él al que se refieren todas nuestras actividades intelectuales, tanto de orden práctico como teórico" ${ }^{38}$ Por tanto, si nosotros queremos describir y analizar el mundo perceptivo, no queda más remedio que "tomarlo - dice Gurwitsch - tal como se ofrece de hecho a la conciencia natural de la vida de todos los días", es decir, tal como se nos ofrece con carácter previo al mundo del pensamiento idealizante $;^{39}$ tal como se nos ofrece en nuestra vida perceptiva.

Pues bien, precisamente es éste un punto de principal conexión entre Husserl y Goldstein. Y también de conexión entre Gurwitsch con ambos pensadores, como reflejan sus cursos y ensayos.

Permítanme citar unas palabras textuales del propio Gurwitsch como ayuda para ir precisando un poco más esta temática.

En sus últimos escritos, Husserl situó en primer plano el "mundo de la vida" (Lebenswelt), es decir, el mundo tal y como es dado en una experiencia de percepción pura, como la base y el fundamento sobre el que se desarrollan las capacidades de la razón, de la razón lógica, matemática y de las ciencias naturales en el sentido específicamente mođerno. Para una interpretación radicalmente filosófica del sentido de estos beneficios de la razón hay que volver al mundo de la vida. Éste presenta formas de organización determinadas. Husserl habla de un 'a 
priori universal del mundo de la vida' a diferencia de un 'a priori objetivo' de las ciencias matemáticas y de otras ciencias formales, el cual se funda en el primer a priori y procede de éste gracias a 'beneficios idealizantes' específicos. Se puede expresar como que en el mundo de la vida está vigente y domina un tipo determinado de lógica que no es la lógica en el sentido propio de una disciplina explicitamente demasiado sistematizante y formal, sino que forma sólo su célula germinal y que por eso puede designarse de forma no inapropiada como 'protológica, ${ }^{40}$

¡Para Husserl, en el mundo de la vida está ya presente una "protológica"! Es evidente que estas palabras escritas por Gurwitsch casi al final de su vida enlazan con aquéllas otras citadas más arriba sobre la necesidad de recuperar la experiencia antepredicativa. En esta experiencia antepredicativa todo sujeto humano ya es capaz de organizar el mundo de un modo natural y espontáneo, como Husserl reflejó en Experiencia y juicio. Hoy diríamos -por lo menos me atrevo yo a plantearlo así en vista de los conocimientos que hoy nos brindan distintas ciencias - que se trata de un mecanismo inserto en nuestra biología de animales humanos - un mecanismo puramente biológico - y sin el cual no seríamos capaces de comprender el sentido de lo que nos rodea en nuestro Umwelt y, por tanto, tampoco de reaccionar adecuadamente ante él, esto es, emitiendo una respuesta con sentido; porque para sobrevivir, como animales que somos necesitamos en todo momento que el mundo no se nos presente caótico, sino con sentido al que nosotros, recíprocamente, podamos también dar una respuesta con sentido. Pero Husserl, evidentemente, se refiere a este mecanismo biológico a su manera, como un "a priori", es decir, como una estructura consustancial a la percepción humana, es decir, como una especie de "lógica" "proto-lógica" que entra en funcionamiento en cada acto perceptivo y que nos permite organizar nuestra comprensión y nuestra respuesta sin tener que pasar por un proceso de aprendizaje; más bien surge en todos y en cada nuevo sujeto de un modo natural y espontáneo. En su ensayo "Acerca de una raíz perceptiva de la abstracción" manifiesta Gurwitsch su afán de dar con esas estructuras de la percepción que -dice él- están en el origen de los procesos de abstracción, tanto generalizadora como formalizadora (el de las formas lógicas). Y para ello confiesa que sus análisis se van a inspirar en este principio de Husserl de que "es en las estructuras de la vida perceptiva en donde es preciso buscar el origen de las formas lógicas", ${ }^{41}$ es decir, el origen de lo que entendemos por pensamiento conceptual, formal o teórico. $\mathrm{O}$, visto desde el otro ángulo: percibir es ya comprender e interpretar pre-racionalmente, y con

40 "Einleitung" a Kurt Goldstein. Selected Papers / Ausgewählte Schriften, p. XXIII. Cursivas mías. Por cierto, Gurwitsch, al mencionar esta "Proto-Logik" deja ver, en nota a pie de página, su agradecimiento a Lester Embree por haber creado esta expresión.

41 "Sur une racine perceptive de l'abstraction", Actes du XI Congrès International de Philosophie, vol. 2 Louvain, Nauwelaerts, 1953, p. 45. 
carácter inmediato, natural y espontáneo, el mundo que nos rodea. Sólo a partir de esta comprensión pre-racional o ante-predicativa inherente a la percepción de todo sujeto pueden levantarse después los modos racionales con los que operamos en mayor o menor medida como humanos.

\section{III}

Goldstein converge con Husserl al considerar que la experiencia perceptiva entraña en sí misma una comprensión organizadora y dadora de sentido, es decir, constituyente, que no es una comprensión conceptual o teórica. Ésta es la idea que hace de corazón de toda la psicología de la Gestalt. Ahora bien, esta comprensión y esta lógica "proto-lógica" que opera en los sujetos, está, según Goldstein, alterada en aquellos que, a causa de sus lesiones cerebrales, perciben el mundo desde la que llama una actitud "concreta" (konkreter) y no desde la que llama la actitud "categorial" (kategorialer) -"proto-lógica"- de los sujetos normales.

Sus investigaciones con pacientes que sufren patologías derivadas de lesiones cerebrales, le permiten descubrir a Goldstein, ciertos constituyentes que, como organizadores naturales y apriorísticos, se hallan en el origen de toda percepción en un sujeto normal. Pero los descubre en el sentido negativo de la ausencia ya que son éstos precisamente los que sus pacientes (los cuales se mueven en una "actitud concreta") no tienen, o los que tienen pero de un modo tan debilitado que terminan por alterar el normal funcionamiento de la percepción (o sea, el propio de una "actitud categorial" - categorial en el sentido goldsteniano que es siempre "proto-lógico" o "pre-racional").

Estos constituyentes cuya ausencia o alteración patológicas descubre Goldstein son - según Gurwitsch - un trascendental hallazgo que confirma los descubrimientos de la fenomenología husserliana. Y sin duda alguna, éste es un punto de conexión muy importante entre Husserl, Gurwitsch y Goldstein.

En nuestra vida normal, como sujetos normales, estos constituyentes de los que hablan estos tres autores actúan en nosotros desde nuestro nacimiento y su carácter es tan universal y tan corriente y espontáneo que lo cierto es que se nos pasan desapercibidos y es difícil dar con ellos y tematizarlos. Pongamos una metáfora aproximativa. La experiencia de las personas que, teniendo algún defecto en la vista, lo subsanamos utilizando gafas o lentillas es que en todo momento gracias a ellas vemos bien, pero sin ser conscientes en todo momento de que si vemos bien es gracias a ellas. Sencillamente lo que experimentamos es que vemos bien y, en esta experiencia, tales artilugios están actuando como la estructura invisible, que no vemos, pero que nos permite la visibilidad del mundo que vemos. La metáfora no es del todo acertada ya que aquí se trata de usar esas gafas o lentillas como un artilugio que no es natural sino producido por nuestra ciencia y tecnología y acoplado a nuestro cuerpo a 
modo de apéndice artificial, mientras que esas estructuras que están ejecutándose en toda percepción de un sujeto normal son un mecanismo apriorístico natural, consustancial a nuestra humana animalidad. Sin embargo, como tal metáfora puede valernos para aproximarnos a la idea que aquí se quiere transmitir: al realizar "un análisis detallado de cada fenómeno [patológico] particular en sus relaciones con la personalidad total del enfermo y con la situación particular del momento", Goldstein se da cuenta de que estos sujetos carecen, como acabamos de señalar, de ciertas estructuras constituyentes que, por el contrario, sí están operando en la percepción de todo sujeto normal.

No puedo hacer aquí referencia detallada de las observaciones descriptivas que hace Goldstein (junto con su colaborador Gelb) de sus pacientes con secuelas de lesiones cerebrales y sin estas observaciones ilustrativas es más dificultoso comprender cómo elabora Goldstein su novedosa teoría. Pero sí puedo ir de modo directo a aquello que Gurwitsch recoge del trabajo de Goldstein con estos pacientes - en realidad, es a esto a lo que necesariamente tenemos que llegar en nuestro recorrido. Y lo que Gurwitsch más enfatiza del trabajo goldsteniano es su distinción ${ }^{43}$ entre actitud "concreta" y actitud "categorial".

Toda la actividad de los pacientes con los que trabaja Goldstein -es decir, todo lo que piensan, sienten y hacen- está dominada por la que llama "actitud concreta". El sujeto se deja guiar pasivamente por la situación presente en la que se encuentra, como si ésta le determinara y le atara de un modo absoluto; hasta el punto de que es incapaz de salir de ella y de poner distancia entre él y ese mundo exterior en el que está en ese momento. Es incapaz de ver más allá de la situación presente y mucho menos de ser libre frente a ella; libre en el sentido de poder distanciarse tomando en cuenta otras posibilidades y eventualidades y de decidirse a la vista de ellas. No; no tiene iniciativa personal ni espontaneidad. Le falta, pues, la creatividad. ${ }^{44}$ En su actitud pasiva se deja guiar por el mundo exterior reaccionando tan sólo cuando este mundo le incita a hacerlo, como un autómata. Por ejemplo, el enfermo no habla más que cuando se le dirige la palabra; nunca toma él la iniciativa de hablar. Y, en general, evita todas las situaciones nuevas que supondrían para él ponerse en otros puntos de vista; al contrario, tiende a realizar una actividad monótona sobre la cual tiene ya un cierto control. Y, algo muy importante: el sujeto per-

42 "Si es verdad que los fenómenos patológicos son modificaciones regulares de hechos normales... no se podrán obtener de los primeros conclusiones relativas a los segundos más que si se han descubierto las leyes de estas modificaciones. Será para esto necesario, en principio, no partir de hechos separados, interpretados aisladamente, como con frecuencia se ha hecho, sino comenzar por observar y por comprender el propio fenómeno patológico como un síntoma revelador de la estructura modificada de la personalidad del enfermo...Esto exige un análisis detallado de cada fenómeno particular en sus relaciones con la personalidad total del enfermo y con la situación particular del momento". Ibid., pp. 283 y 285.

44 Cfr. "Le fonctionnement de l'organisme d'après K. Goldstein", pp. 130s, 136s. 
manece preso en un contexto de acción práctica exclusivamente y no consigue distanciarse de él. Se mantiene en una actitud que "consiste esencialmente en saber manejar perfectamente las cosas (y el lenguaje...) y utilizarlas en una situación dada para tales o tales otros fines prácticos, pero sin poder mirarlos, contemplarlos, hacerse puro espectador con respecto a ellas, poner una distancia entre sí mismo y el mundo...Para que un objeto tenga, a sus ojos, un sentido y para que (el sujeto sepa) hacer alguna cosa, es indispensable que este objeto se presente con un carácter de realidad inmediata que provoque...una reacción práctica. Para (él) solamente existe lo que está integrado, con un valor funcional determinado, en una situación de acción global [concreta]". ${ }^{45}$ Es decir, los asuntos que llegan al sujeto son definidos nada más que por su finalidad y por su uso práctico y esto siempre bajo esas circunstancias concretas en las que se halla.

Por el contrario, en un sujeto normal, sano, prevalece la "actitud categorial" ("proto-lógica"). Para este sujeto, la situación en la que está inmerso está como enmarcada en un contexto más amplio y la concibe como cambiante. Por supuesto que en su vida cotidiana se comporta continuamente en referencia a una $\mathrm{u}$ otra situación concreta, pero contemplará la situación como poniéndose mentalmente a distancia, y la observará desde otros puntos de vista sin que esto le cause ningún problema. Esto le permite tener en cuenta otras posibilidades que en la situación presente no se están dando de hecho. Por ejemplo, puede prever efectos deseados o indeseados que están fuera de esa situación tal como se presenta en ese momento pero que se podrían producir. Puede posicionarse ante la situación dada, y teniendo en cuenta esos efectos actuar con iniciativas y tomar decisiones en vista de las posibilidades de acción a realizar, poniendo en marcha aquellas acciones que modificarán esta situación en la que se halla a fin de evitar esos efectos secundarios indeseados o poniendo en marcha aquellas otras que traerían consigo los efectos deseados. $\mathrm{Y}$ por supuesto que le es posible tener en cuenta, simultáneamente, distintos aspectos de la situación, relacionarlos entre sí y valorarlos antes de decidir el modo en el que actuará. Para esto se vale de su capacidad para tener unos aspectos destacándose en primer plano y otros en segundo, tan sólo sirviéndole de fondo. El sujeto es capaz de desligar los objetos y los distintos aspectos de su situación concreta del vínculo que mantienen con ella y pasar a referirlos, pues, a un orden abstracto, etc. En otras palabras, el sujeto normal, en su actitud "categorial" normal, consigue ejercer la función de "objetivación" y "tomar distancia", como sujeto, frente a la realidad dada puesto que nunca permanece reducido a ninguna situación concreta en la que se halle en cada momento; de este modo, está en condiciones de desarrollar su propia iniciativa,

45 A. Gurwitsch "Psychologie du langage", Revue Philosophique de la France et de l'Étranger, CXX (1935) pp. 408s. 
tanto a nivel de pensamiento como de acción. ${ }^{46}$ Este sujeto es, por tanto, para Goldstein, un sujeto "libre". Es decir, no está supeditado a ninguna realidad inmediata. Y enfrenta las situaciones novedosas e inesperadas como un reto ante el cual es capaz de soportar, incluso, esa cierta dosis de angustia que siempre lleva consigo este tipo de situaciones. ${ }^{47}$

\section{IV}

Planteemos ahora la cuestión de cuáles son esas estructuras constituyentes que están funcionando con toda normalidad en los sujetos sanos y que, por el contrario, han dejado de funcionar en los sujetos estudiados por Goldstein con lesiones cerebrales. Y abordemos esta cuestión junto con la de en qué sentido hay coincidencia entre lo que sobre esto nos dice Goldstein y lo que se nos dice desde la fenomenología husserliana asumida, a su vez, por Gurwitsch ${ }^{48}$. En sus escritos y en sus cursos, Aron Gurwitsch trabaja incesantemente sobre estas estructuras de la percepción, es decir, sobre cómo se cons-

46 Cfr. A. Gurwitsch "Einleitung" a Kurt Goldstein. Selected Papers / Ausgewählte Schriften, pp. XVIII-XX. Para ver el contraste entre la actitud "concreta" y la "categorial" puede ser muy útil el excelente comentario que hace Alfred Schütz en el cap. 10 de Collected Papers vol I. The Problem of Social Reality, The Hague, M. Nijhoff, 1962, libro traducido al castellano como El problema de la realidad social, Buenos Aires, Amorrortu, 1974. Cfr. "Le fonctionnement de l'organisme d'après K. Goldstein", p. 138. En su libro Trastornos del lenguaje. Las afasias. Su importancia para la Medicina y la Teoría del lenguaje (Barcelona, Editorial Científico Médica, 1950), afirma Goldstein que la actitud categorial o abstracta "es fundamental para las siguientes funciones: 1. Asumir voluntariamente una situación mental, tomando la iniciativa incluso al comenzar una función que se nos ha exigido. 2. Pasar voluntariamente de un aspecto de la situación a otro, eligiendo. 3. Recordar simultáneamente varios aspectos de una situación, reaccionar frente a dos estímulos que no aparecen intrínsicamente unidos. 4. Asimilar la parte esencial de un conjunto, dividiéndolo en diferentes partes, aislándolas voluntariamente y combinándolas luego en el conjunto. 5. Considerar de un modo abstracto propiedades comunes, proyectar de antemano, adoptar una actitud con referencia a lo 'posible' y pensar o ejecutar de un modo simbólico. 6. Deslindar el ego del mundo circundante. Todas estas funciones - dice- aparecen en los enfermos más o menos alteradas" (p. 8).

Nos referimos exclusivamente a enfermos con anomalías en su percepción y comportamiento fruto de sus heridas en el cerebro. Aquí no nos referimos a esos otros sujetos que han sufrido la pérdida de algún miembro. El caso de estos sujetos es muy diferente y requiere otro tipo de explicación para su comportamiento; un comportamiento que no es patológico sino del que simplemente el psiquiatra quiere tener más comprensión. Por ejemplo, el comportamiento de un sujeto que ha sufrido la amputación de uno de sus brazos y que, pese a ello, sigue "contando con" ese brazo y abierto a los movimientos que antes ejecutaba con él. Non hay ninguna patología aquí. Ni hay tampoco ausencia de estructuras apriorísticas constitutivas sino todo lo contrario. Es gracias a ellas, intactas en él, por lo que el cuerpo actual de este sujeto sigue abierto a aquellos movimientos que antes podía realizar. 
tituye y sobre qué es lo que interviene en todo noema perceptivo ${ }^{49}$. Como fenomenólogo conoce bien el alcance de la aportación de Husserl sobre la experiencia perceptiva. $\mathrm{Y}$ es desde su orientación fenomenológica como va a las investigaciones de Goldstein y como encuentra que ambos autores, por separado, hablan de las mismas estructuras y en muy parecidos términos: Como esos constituyentes tan indispensables en todo sujeto para poder organizar con normalidad su experiencia perceptiva. Sólo que uno se refiere a ellas como las que todos poseemos en cuanto sujetos perceptivos normales, y el otro, en cumpliendo su papel de neuropsiquiatra, se refiere a ellas como las grandes ausentes en la anómala actividad perceptiva de sus pacientes $\mathrm{y}$, por tanto, en su también anómalo comportamiento. ${ }^{50}$

De sus descripciones clínicas acerca estos pacientes, Goldstein diagnostica que falta en ellos, o que está muy debilitada, la estructura de diferencia-

49 Todo el trabajo investigador y docente de Gurwitsch está volcado en realizar una fenomenología de la percepción y en analizar cuáles son y cómo funcionan estas estructuras constitutivas con las que conseguimos organizar cada nuevo noema perceptivo. Probablemente su libro The Field of Consciousness (El campo de la conciencia. Un análisis fenomenológico, Madrid, Alianza Editorial, 1979), escrito en 1953, en la madurez de su vida intelectual, es el mejor libro que se haya escrito nunca sobre fenomenología de la percepción. Es un libro en el que va analizando, uno por uno, los componentes constitutivos del acto perceptivo. Para ello se vale de los descubrimientos de la fenomenología husserliana y de los de la psicología gestaltista y de Gelb-Goldstein. El estilo es un estilo de análisis sistemático que permite a quien lee el libro avanzar ganando en comprensión fenomenológica sin perderse en ningún momento. Como merleaupontyana que soy, me atrevo a decir que Phénoménologie de la percepción, aunque escrito en 1942, debería leerse únicamente después de The Field of Consciousness. Como hemos indicado antes, propiamente The Field es anterior a Phénoménologie de la perception ya que aunque aquél haya sido publicado más tarde que éste, en realidad, en él Gurwitsch aborda con extensión esta temática que siempre había formado el núcleo de su interés y que ya él había tratado desde 1928 en todos sus ensayos anteriores y en sus cursos como docente, si bien con menos extensión. Cuando Gurwitsch lee Phénoménologie de la perception, estando ya en E.E.U.U., le hace estos comentarios a su amigo Alfred Schütz en carta del 11 de agosto de 1947: "Ich lese jetzt Merleau-Ponty's "Perception". Ich höre aus dem Buch enorm viel aus meinen Vorlesungen heraus. Er hat viel von mir gelernt und viel übernommen. Nicht nur in den Einzelheiten, wo er manches entwickelt hat. Ich zweifle daran ob er ohne meinen Einfluß auf die Idee gekommen wäre, das psycho-pathologische Materiel phänomenologisch auszudenten. Meine Stimmung bei die Lektuere ist eine Mishung aus Freude und Melancholie. Ehrliche Freude über das gelungen Buchm das wirchlich eine schöne Leistung ist; und Freude auch darüber, daß mein Einfluß dabei in einem gewissen Sinne Pate stand. Es ist shon ein schönes Gefühl zu wissen, daß meine Pariser Jahre nicht für das Nichts waren, and daß etwas bei menen Vorlesungen heraus gekommen ist. Und die Melancholie bezieht sich auf den Modus praeteritus. Nie werde ich hier eine solche Wirkung haben". Alfred Schütz Aron Gurwitsch Briefwechsel 1939-1959 (ed. Richard Grathoff), W. Fink, 1985, p. 158.

50 "When we refer to psychopathological findings, it is because, by way of contrast, these findings permit us to disclose conditions which, justo $\mathrm{n}$ account of their realization in the case of normal persons, are likely to be taken for granted and hence to be overlooked". Con estas palabras cierra Gurwitsch su ensayo "Gelb-Goldstein's Concepto of 'Concrete' and 'Categorial' Attitude and the Phenomenology of Ideation". 
ción figura-fondo. Con lo cual, la función de objetivación también se ve afectada puesto que es inexistente la conciencia de horizonte externo en estos sujetos. Tales carencias afectan a todo el organismo y dan lugar a una patológica relación con el mundo.

Para los sujetos normales, cada objeto percibido es percibido situado en un "campo perceptivo" y no puede ser percibido como tal si no es dentro de tal campo. Esta idea la vemos en Husserl en sus Ideas de $1913^{51}$ y de todos los fenomenólogos ha sido Aron Gurwitsch quien más se dedicó al estudio de este fenómeno. ${ }^{52}$ A su vez, el campo perceptivo es una de las ideas-eje de la psicología de la Gestalt así como del neuropsiquiatra Goldstein. ${ }^{53}$ Será el gestaltista Edgar Rubin quien, partiendo de ella, en 1915 establezca la diferencia que llamó "figura"-"fondo": toda percepción de un objeto supone que éste destaca, como "figura" (Gestalt), sobre un "fondo" perceptivo inarticulado. Es este "fondo" el que Husserl denominaba "campo perceptivo": el objeto de la atención perceptiva nunca se ofrece solo sino que se destaca como tal sobre un fondo que está ahí pero únicamente co-presente; este fondo co-presente es el "campo perceptivo", es decir, un campo perceptivo-práxico. No forman parte del fondo todos los hechos que "objetivamente" se encuentran entorno a la figura percibida de la que se hace "tema" de atención, sino sólo los que le aparecen de algún modo al sujeto. Es decir, no se trata de los hechos que podrían estar copresentes para el sujeto, sino de los que lo están en realidad. En otras palabras, el entorno perceptible puede no coincidir con el campo temático (por ejemplo, un artesano ve en un escaparate una herramienta de la que ya conoce su empleo práctico; esto ocurre porque dicha herramienta tiene para él valores funcionales que él obtiene de una situación no perceptible en su momento actual). Cuando el sujeto atiende a lo que hasta ese momento sólo se estaba dando como copresente, la percepción se reorganiza es ese mismo momento y esto pasa a ser el centro del nuevo campo temático; y, a la vez, el dato que antes ocupaba su atención, si es que no lo pierde de vista pasaría a formar parte del nuevo fondo, segundo plano u "horizonte" exterior. Y para la conciencia perceptivo-práxica, todo lo copresente es susceptible de transformarse

51 Cfr. $§ 27$ de Ideas, y cfr. La crisis de las ciencias europeas y la fenomenología trascendental, pp. 111 s y 171 .

52 Esto lo evidencia el título de su libro The Field of Consciousness, El campo de la conciencia. Pero realmente es tema que ocupa buena parte de su obra. Por ejemplo, cfr. Esquisse de la phénoménologie constitutive (sobre todo, Cap. II, §6 y Cap. III, §7). Por ejemplo, en "Thème et attitude" (que está publicado como Apéndice II en Esquisse y procede de una conferencia de 1934-35) habla del "tema" en cuanto centro de un campo de significación: el campo de la conciencia. Y en el libro póstumo Marginal Consciousness (Athens, Ohio University Press, 1985) habla de aquello que forma el "margen" del tema.

Cfr. "L'analyse de l'aphasie et l'étude de l'essence du langage". Es muy conocido el caso de los pacientes de Goldstein que aunque han perdido la visión de media retina son capaces de reorganizar su visión porque, según él, cada objeto percibido está situado en un campo perceptivo-práxico. 
en centro temático, y viceversa. ${ }^{54}$ Por tanto, lo que percibimos necesariamente lo percibimos dentro de esta especie de "proto-lógica" perceptiva que funciona en el sujeto organizando la realidad que vive de un modo inmediato y natural o como figura o "tema", o como fondo o "campo" temático; y siendo, pues, inevitable que esta realidad se transforme continuamente y se produzca el fenómeno de la alternancia entre tema y campo, figura y fondo.

Este descubrimiento teórico por parte de Husserl queda confirmado con las observaciones empíricas de Goldstein. Este neuropsiquiatra nos muestra que el daño neuronal de sus pacientes trae como consecuencia final que, en vez de funcionar en ellos la necesaria diferenciación perceptiva entre entre "figura" y "fondo", "figura" y "fondo" están nivelados; o, lo que es lo mismo, los pacientes no consiguen llevar a término el proceso de centralización. Esta nivelación, fruto de una centralización imperfecta o inexistente les impide actuar con normalidad y quedar reducidos a la actitud "concreta" antes descrita. La reacción pasiva que ellos manifiestan ante la situación concreta en la que encuentran proviene de que, por su daño cerebral, no se pone en marcha (o está muy debilitada) la facultad de "objetivación". Y esta facultad caracteriza, según Goldstein, nuestra conciencia humana. La facultad de "objetivación" consiste en poder distanciarse del mundo para verlo en sus posibilidades diferentes, como en perspectiva. Y en esta perspectiva, unos objetos aparecen para el sujeto en primer plano (como "figura") y otros en segundo plano (como "fondo"), al igual que en los objetos perceptivos que son para él "figura" se establece una distinción entre lo que es esencial en ellos (su eidos) y lo que no lo es. Y, como consecuencia, el sujeto prestará su atención a los primeros y no a los segundos y sabrá atender a unos elementos y dejar de lado otros. ${ }^{55} \mathrm{Sin}$ embargo, su imposibilidad de organizar o estructurar su mundo conforme a un proceso de centralización (Zentrierung) y, a la vez, de objetivación y distanciamiento, se traducirá en el paciente en una falta de precisión y de exactitud en sus acciones. Y su imposibilidad de conocer los objetos que percibe según su características esenciales y para practicar la "variación libre" a partir del eidos de esos objetos (por medio de la cual lo realmente existente se desposee de su realidad, de sus determinaciones espacio-temporales de las que se deriva su carácter individual y de todos sus demás caracteres existenciales) le impide al paciente concebir esos objetos bajo otras posibilidades, imaginarlo en experiencias diferentes. ${ }^{56}$ Así, el enfermo se verá perdido e incapaz de responder a

\section{Cfr. cap. III, § 8 de Esquisse.}

55

56

Cfr. "La science biologique d'après M. K. Goldstein", p. 400.

Cfr. "Gelb-Goldstein's Concept of 'Concrete' and 'Categorial' Attitude and the Phenomenology of Ideation ", parte III. El paciente tiene delante madejas de lana de distintos colores. La diferencia goldsteniana entre la actitud "concreta" y la "categorial" es, dice Gurwitsch, "la diferencia que existe entre percibir una cosa según el carácter típico que posee y aprehenderla en cuanto representante del tipo en cuestión...'Coger juntas...las madejas de igual brillantez en virtud de la conformidad de hecho que existe entre ellas...es muy diferente de saber que 
las exigencias que vienen del medio normal en el que vivimos los humanos; $y$, en el caso de ser enfrentado a ellas, sin duda, al no saber responder adecuadamente, caerá en una reacción catastrófica tras otra con la consiguiente angustia que esto entrañará para él. Ante un estímulo exterior, o bien se ve dificultada su capacidad de reaccionar o, si el estímulo hace efecto en él, la reacción producida puede ser de una intensidad fuera de lo normal. ${ }^{57}$

En mi opinión, la estructura de horizonte temporal interna, en el sentido en que la entiende Husserl, también se halla alterada en los pacientes de Goldstein. Para los pacientes su mundo se ha reducido a la pura actualidad. No hay en ellos anticipaciones con respecto a actos perceptivos futuros. ${ }^{58}$ No se sienten libres para anticipar y acceder a otras percepciones y perspectivas diferentes a las que tienen en el momento presente. $Y$, por consiguiente, tampoco tendrán ningún motivo para iniciar algún movimiento, algún cambio de postura de su cuerpo, que les permitiría acceder a esas otras percepciones copresentes en su percepción actual bajo la forma de implicaciones potenciales. No están copresentes. Sin embargo, Gurwitsch concentra su atención en las modificaciones que sufre la estructura "figura"-"fondo"que acabamos de analizar para mostrar que Goldstein avala desde su campo de saber científico la idea filosófica de Husserl de que organizamos nuestra experiencia perceptiva sin que tenga que intervenir para ello ningún pensamiento categorial externo a la propia experiencia perceptiva inmediata. Su amigo Alfred Schütz, volviendo sobre el estudio sobre la afasia de Goldstein hace una reflexión muy interesante sobre la estructura de horizonte interno en los pacientes que complementa el análisis de Gurwitsch en similar sentido. ${ }^{59}$ Pero hablar de esto ahora excedería el propósito trazado en esta ponencia. Será asunto a abordar en otra ocasión.

\section{ABSTRACT}

Aron Gurwitsch es el primero que hace en Francia, como fenomenólogo, una exposición profunda y sistemática de la fenomenología husserliana. Desde el comienzo él centra su investigación en una fenomenología de la percepción. Los aspectos en los que más focaliza su atención le conducen, ya desde el inicio de su labor investigadora, a sentir una profunda sintonía con el trabajo del neưropsiquiatra Kart Goldstein. Él también el primero que hace en Francia una divulgación del trabajo

tales madejas sean similares (o análogas) en virtud de la brillantez y de seleccionar dicha propiedad como principio clasificatorio' ", dice Gelb. Cfr. El campo de la conciencia, p. 279. Cfr. "Le fonctionnement de l'organisme d'après K. Goldstein", passim, y "Einleitung ", pp. XII-XVI.

58 Gurwitsch expone así el pensamiento husserliano de la conciencia de horizonte interno en Esquisse, en cap. III, § 3 y 7, en cap. IV, § 5 y 6 , y en cap. IV, § 9 y 10. 
de este neuropsiquiatra desde la filosofia. De modo que en la obra de Gurwitsch hay dos líneas de investigación que se juntan y que están en él desde el principio: su contacto con el Husserl de Friburgo y su profunda compenetración con él, y su trabajo en Frankfurt con Goldstein y con el psicólogo de la Gestalt que colabora con él: Adhémar Gelb. En esta ponencia intento buscar alguna respuesta a la cuestión de qué ve Gurwitsch en el análisis que hace Goldstein de sus pacientes con lesiones. cerebrales para considerar él que este análisis goldsteniano es extremadamente coincidente con el propio análisis fenomenológico de la percepción de Husserl. 
